\title{
Exploring Data Analytics of Data Variety
}

\author{
Tiago Cruz, Jorge Oliveira e Sá and José Luís Pereira \\ ALGORITMI Research Center, University of Minho, Guimarães, Portugal \\ a66785@alunos.uminho.pt; \{jos, jlmp\}@dsi.uminho.pt
}

\begin{abstract}
The Internet allows organizations managers access to large amounts of data, and this data are presented in different formats, i.e., data variety, namely structured, semi-structured and unstructured. Based on the Internet, this data variety is partly derived from social networks, but not only, machines are also capable of sharing information among themselves, or even machines with people. The objective of this paper is to understand how to retrieve information from data analysis with data variety. An experiment was carried out, based on a dataset with two distinct data types, images and comments on cars. Techniques of data analysis were used, namely Natural Language Processing to identify patterns, and Sentimental and Emotional Analysis. The image recognition technique was used to associate a car model with a category. Next, OLAP cubes and their visualization through dashboards were created. This paper concludes that it is possible to extract a set of relevant information, namely identifying which cars people like more/less, among other information.
\end{abstract}

Keywords: sentimental and emotional analysis, machine learning, data analysis techniques.

\section{$1 \quad$ Introduction}

With the increasing use of the internet by people and organizations, the amount of information has grown exponentially. The digital universe presents a diversity of data such as social networking data, RFID sensor data, geospatial data, website data, mobile device data, among others. This data presents a different data formats, i.e., variety, and can be classified as structured, semi-structured or unstructured data.

Structured data has a rigid structure, usually stored in databases. Unstructured data does not have a defined structured, and it can be for example text, videos, audio files, photographs, among others [1]. Semi-structured data has a flexible structure, containing a set of tags or markers, and can be found in CSV, XML, or JSON files [2].

The data analysis can bring interesting information to organizations [3]. Usually, only $5 \%$ of the data belongs to structured format, when the remaining $95 \%$ are semi-structured or unstructured, and normally these data are not analyzed [1]. This reveals that there is a large amount of data that should be analyzed to understand its potential, which is not the case in most organizations.

The data analysis with data variety can be an important aspect for organizations, as it can offer relevant information to organization managers, facilitating the decision-making process. 
There are different data analysis techniques capable of extracting information relevant to decision-making processes. It is considered that structured data analysis techniques are already mature and validated by the scientific community and are widely used by organizations, but this is not true for semi-structured and unstructured data.

The objective of this paper is to understand if it is possible to extract information insights from this data variety, i.e., unstructured and semi-structured data.

This paper is based on an experiment, using datasets composed of semi-structured and unstructured data. Data analysis techniques were applied according to the data formats and the objective is to be able to extract information from these data.

This paper is structured as follows: Section 2 presents the related work, i.e., several data analysis techniques are presented for semi-structured and unstructured data; In section 3 the experience is described, describing in detail the datasets, the tools and techniques used, the architecture of the solution developed and the results of the experiment test; In section 4 the results obtained are discussed through data analysis presented with dashboards; Finally, in section 5, conclusions, limitations and future work are presented.

\section{Related Work}

One of the concerns of this work was to identify/leverage analytical techniques that can be used in semi-structured and unstructured data. Tables 1 and 2 identify some studies that use data analysis techniques and which data types are used.

Table 1. semi-structured data techniques

\begin{tabular}{lll}
\hline Reference & Techniques & Data type \\
\hline$[4],[15],[16]$ & Natural Language Processing & text \\
{$[4]$} & HTML Trees (DOM) & text \\
{$[5],[6]$} & Link Prediction & Collaboration Networks; So- \\
& & cial Networks; Infrastructure \\
& & Networks; Sports Networks; \\
& & Biology Networks \\
{$[7]$} & Probabilistic Three-way Entity Model (TEM) & text \\
{$[8]$} & Entity Linking & Entities (text) \\
{$[9],[10],[11]$} & Naive Bayes Classifier & Text and numeric values \\
{$[12]$} & Multilayer Perceptron, (MLP) & Time series \\
{$[13]$} & Entity Recognition & text \\
{$[13],[14]$} & Sentiment Analysis & text \\
\hline
\end{tabular}

Table 2. unstructured data techniques

\begin{tabular}{lll}
\hline Reference & Techniques & Data type \\
\hline$[4],[15],[16]$ & Natural Language Processing & text \\
{$[17],[18]$} & Topic Modeling Probabilistic & text \\
{$[19]$} & Latent Semantic Visualization & Time-stamps \\
{$[20]$} & First Story Detection & text \\
{$[21]$} & Event Extraction & Entities (text) \\
{$[22]$} & Data Discovery & text \\
{$[13]$} & Entity Recognition & text \\
{$[13],[14]$} & Sentiment Analysis & text \\
{$[23]$} & Image Recognition & image \\
\hline
\end{tabular}


The techniques described in the tables depends on the characteristics of the data types used in the identified studies.

In the following section, the techniques used are Natural Language Processing, as well as Sentimental and Emotional Analysis for comments about cars and the Image Recognition Technique to analyze car images.

\section{Experiment Description}

The experiment will use a dataset with two data formats as described in section 3.1. The tools used are presented in section 3.2. In Section 3.3, the solution architecture is described. Finally, the solution is tested with a set of test data and the results obtained are evaluated.

\subsection{Datasets Used}

The dataset used in this experiment, was used in a study on classification of entities based on opinions and it consists of 3 years of comments on cars, namely 2007, 2008 and 2009 [24]. This dataset has up to 250 cars models per year. The dataset content is divided between the fields: author, date, and the textual opinion about the car. The data format inserted in this dataset, are considered semi-structured.

In addition to the dataset referred above, a dataset containing car images from car models of the year 2008 was included. This dataset is considered unstructured data.

\subsection{Technologies and Tools Used}

In this section, it will be presented the technologies used in this experiment and the associated techniques if applicable, see Table 3.

\subsection{Experiment solution architecture}

In Figure 1 the experiment solution architecture is presented. This architecture aims to extract information from the dataset.

As shown in figure 1, the dataset is composed of comments on cars with the semistructured format and images of cars with the unstructured format.

The image car model used as input data will be classified using Visual Recognition IBM tool. To do this it was necessary to create a custom classifier to be able to recognize which category belongs to each car model.

The Natural Language Understanding tool was used to retrieve the sentimental and emotional analysis from the comments dataset, and to do this it was necessary to use the Watson Knowledge Studio. In the Watson Knowledge Studio tool, it was possible to create a machine-learning model capable of identify a set of patterns relevant to this experiment. 
Table 3. Tools used in the experiment

\begin{tabular}{|c|c|c|}
\hline Tool & Description & $\begin{array}{l}\text { Techniques (if ap- } \\
\text { plicable) }\end{array}$ \\
\hline $\begin{array}{l}\text { Natural Lan- } \\
\text { guage Under- } \\
\text { standing IBM }\end{array}$ & $\begin{array}{l}\text { The purpose was to retrieve a set of information: entities, keywords, } \\
\text { relations, and sentimental and emotional analysis; of the commen- } \\
\text { taries alluding to a car model. }\end{array}$ & $\begin{array}{l}\text { Natural Language } \\
\text { Processing; Emo- } \\
\text { tional Analysis; Senti- } \\
\text { mental Analysis }\end{array}$ \\
\hline $\begin{array}{l}\text { Watson } \\
\text { Knowledge } \\
\text { Studio IBM }\end{array}$ & $\begin{array}{l}\text { In the experiment, this tool was used as a supplement to the Natural } \\
\text { Language Understanding IBM tool, and its objective was to recog- } \\
\text { nize a set of standards through a machine learning model. }\end{array}$ & \\
\hline $\begin{array}{l}\text { Visual Recogni- } \\
\text { tion IBM }\end{array}$ & $\begin{array}{l}\text { The Visual Recognition IBM was used to associate an image with a } \\
\text { car category, and this was possible using a custom classifier for the } \\
\text { categories that were intended to be recognized. }\end{array}$ & Image Recognition \\
\hline Postman & $\begin{array}{l}\text { The Postman was used to perform POST requests for the Visual } \\
\text { Recognition (to classify a car model into a category), and the Natu- } \\
\text { ral Language Understanding (for information extraction about com- } \\
\text { ments associated with a car). The information obtained was saved } \\
\text { in JSON format. }\end{array}$ & \\
\hline SQL Server & $\begin{array}{l}\text { The version used was SQL Server Enterprise Edition } 2014 \text { and } \\
\text { served as a data repository, where the internship database was stored } \\
\text { and the On-Line Analytical Processing (OLAP) cubes. }\end{array}$ & \\
\hline SSIS & $\begin{array}{l}\text { The SQL Server Integration Services (SSIS) are used to develop } \\
\text { some Extraction, Transform and Loading (ETL) functionality. }\end{array}$ & \\
\hline SSAS & $\begin{array}{l}\text { The SQL Server Analysis Services (SSAS) are used to create OLAP } \\
\text { cubes. }\end{array}$ & \\
\hline Talend & $\begin{array}{l}\text { The Talend was used because SSIS did not have the functionality of } \\
\text { handling data in JSON format. Therefore, this tool was used to per- } \\
\text { form ETL tasks, namely transforming JSON format data and insert- } \\
\text { ing into a database. }\end{array}$ & \\
\hline
\end{tabular}

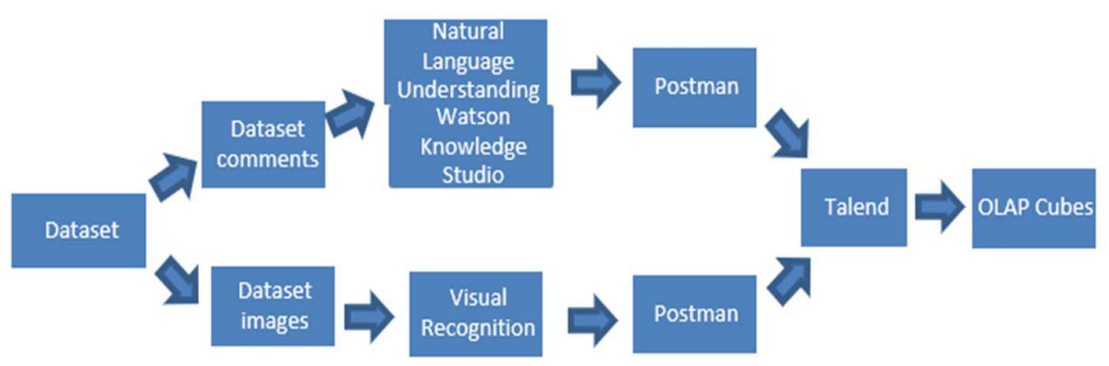

Fig. 1. Experiment solution architecture

As seen in figure 1, the data obtained from the Natural Language Understanding and Visual Recognition tools, are presented in the Postman tool. This tool saves the files in JSON format. To insert these data formats into SQL Server, it was necessary to use the Talend tool, where ETL tasks were performed, and then insert the data into a staging database in the SQL Server tool.

To create the OLAP cubes, it was necessary to use SSIS to define an ETL process to perform a set of transformations on the data, to ensure that they were ready to be 
inserted into the OLAP cubes. In SSAS, three OLAP cubes were created as entities, relations and keywords studied different and unrelated information.

\subsection{Experiment solution testing}

To be able to evaluate the possibility to retrieve information using the experiment, an excerpt of comments and the corresponding images are retrieved from the dataset to understand if the architecture proposed can:

- classify the car image and associate it with a car category; and

- find a set of relations, keywords, entities, sentiments and emotions in the excerpt of comments.

Regarding the dataset of images and to classify a model of a car in a certain category, a machine learning tool was used, using Visual Recognition technique. For this, a classifier was developed that from the image of the car can associate it with a category. Initially, a set of images with the corresponding car category was provided, so the tool can learn how to classify images into categories. To validate this classification, a set of tests was performed, for example, for an image that refers to the "dodge_pickup_3500" model, resulting in a classification in three categories: "Coupe" with a precision of 0.56 , "Pickup_truck "with precision of 0.99 and" Sport_utility "with precision 0.84 . The chosen category was "Pickup_truck", because it obtains the best result.

Regarding the comment dataset, a set of tests was carried out with an excerpt of comments about cars, to extract information from these data. It was possible to identify a set of information as: entities, keywords and relations. This information was possible to obtain, because in the Watson Knowledge Studio tool (associated as a supplement to the Natural Language Understanding tool) exists a machine learning model capable of recognizing a set of patterns. A set of entities and relationships for the created model are identified, to be able to recognize these patterns in the Watson Knowledge Studio tool. A set of eight training files containing excerpts of comments for the model to be able to recognize entities and relationships are used. Next, the machine learning model created in the Watson Knowledge Studio is used in the Natural Language Understanding tool, where associated with the different relations, keywords and entities will recognize a set of information using Natural Language Processing technique and associate to each keyword and entity the sentimental and emotional analysis.

An excerpt of comments on the model "dodge_pickup_3500" is used as a test and the result obtained is shown in figure 2. As shown in figure 2, it was possible to extract a set of information from this data, namely keywords, entities and relations. It was possible to obtain the sentimental analysis through the variable score, and emotional analysis through the variables Emotion_sadness, Emotion_Joy, Emotion_fear, Emotion_disgust and Emotion_anger, of the entities and keywords.

For example, it was possible to identify the entity "Car_Noise" and associate to this entity the variable "score" sentimental analysis with the precision of 0.83 , in which it is identified as being positive. Note this value varies between 1 and -1 . In the keywords was identified for example "dodge Ram" and emotional analysis of that word obtain the following values of emotions: sadness with precision of 0.15 ; joy with precision of 
0.32 ; fear with precision of 0.08 ; disgust with precision of 0.07 ; and anger with precision of 0.12 . Note the range of emotion values varies between 0 and 1 . In relations it was possible to identify the relationship between two distinct entities being "engine" and "Quiet".

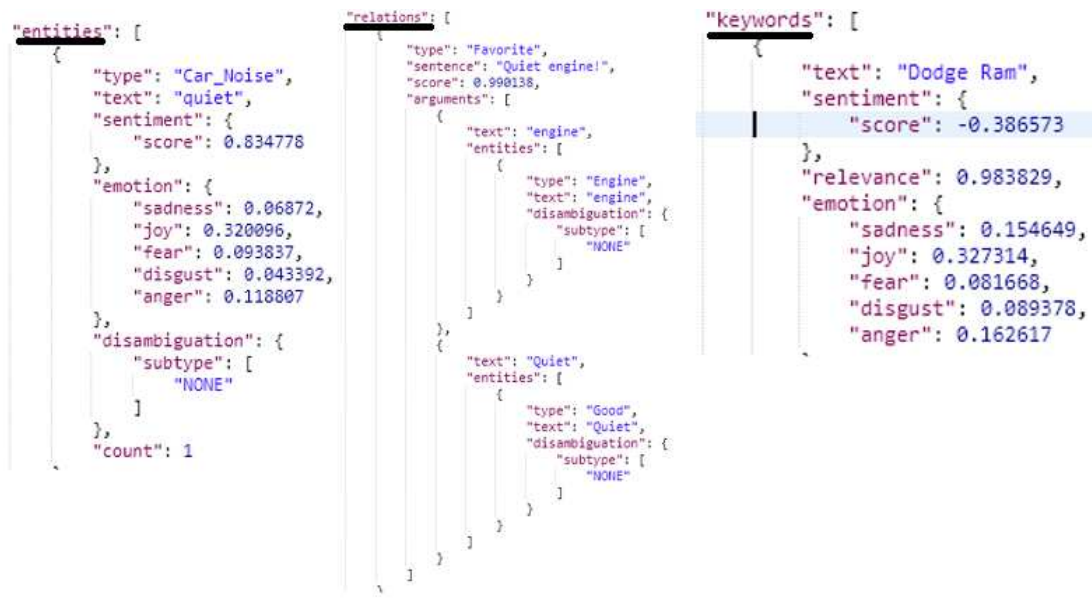

Fig. 2. Entities, relations and keywords

\section{$4 \quad$ Results discussion}

This section presents an analysis performed with the OLAP cubes, and its visualization through dashboards. A dashboard composed of emotional analysis was developed using the Entities cube, followed by a dashboard for sentimental analysis and other features of a car model using the three cubes generated, namely Keywords, Entities and Relations Cube.

Figure 3 presents five types of emotions associated with a set of car models. The choice of these car models is due the fact that a segmentation of data divided as "Car Category 1", "Car Category 2" and "Car Category 3" was created, of the car models that belong to "Car Category 1" "Pickup", "Car Category 2" "Pickup" and "Car Category 3" "Pickup_Truck". For each of the five tables, an indicator value was used to measure the emotion of the car model and a colored mark was also added to understand if the value is acceptable (green color), neutral (yellow color) or bad (red color). The tables that measure the emotions "anger", "sadness", "fear" and "disgust" do not have values with significant impact, so their analysis in this case may not have impact in the decision-making process. The joy emotion has a greater impact than the others, but even so, most car models have a value considered neutral, only the "gmc" car model was below normal.

At last, the figure 4 illustrates a deeper analysis of several factors to a specific car model. In this figure, a set of tables with several factors associated with a car model of the brand "volvo" and model "s40" is presented. 


\begin{tabular}{|c|c|c|c|c|c|c|}
\hline \multirow{2}{*}{$\begin{array}{l}\text { Fear emotion by Brand-model } \\
\boxplus \text { dodge }\end{array}$} & \multicolumn{2}{|c|}{ Hedian_fear } & \multicolumn{3}{|c|}{ Disgust emotion by Brand-model Median_disgust } & Ja \\
\hline & 0 & 0,085 & Đford & 0 & 0,056 & \\
\hline$\boxplus$ chevrolet & O & 0,084 & $\oplus$ chevrolet & o & 0,051 & \\
\hline Đ toyota & o & 0,071 & $\oplus$ toyota & o & 0,047 & \\
\hline$\oplus \mathrm{gmc}$ & - & 0,068 & $\oplus$ dodge & ? & 0,043 & \\
\hline$\boxplus$ nissan & - & 0,067 & $\boxplus$ nissan & - & 0,037 & \\
\hline$\boxplus$ ford & - & 0,064 & $\oplus \mathrm{gmc}$ & ? & 0,034 & \\
\hline$\boxplus$ mitsubishi & - & 0,056 & $\$$ mitsubishi & - & 0,024 & \\
\hline Total Geral & - & 0,073 & Total Geral & 0 & 0,046 & \\
\hline Anger emotion by Brand-model & \multicolumn{2}{|c|}{ Median_anger } & \multicolumn{4}{|c|}{ Sadness emotion by Brand-model } \\
\hline Ðchevrolet & 0 & 0,080 & $\boxplus \mathrm{gmc}$ & 0 & \multicolumn{2}{|l|}{0,157} \\
\hline iford & - & 0,076 & $\boxplus$ dodge & - & \multicolumn{2}{|l|}{0,144} \\
\hline Idodge & - & 0,073 & $\oplus$ toyota & ○ & \multicolumn{2}{|l|}{0,128} \\
\hline$\boxplus$ toyota & 0 & 0,066 & $\boxplus$ ford & 0 & \multicolumn{2}{|l|}{0,128} \\
\hline$\boxplus \mathrm{gmc}$ & - & 0,055 & $\boxplus$ chevrolet & - & \multicolumn{2}{|l|}{0,121} \\
\hline$\boxplus$ nissan & 0 & 0,055 & $\oplus$ nissan & 0 & \\
\hline$\bigoplus$ mitsubishi & 0 & 0,045 & $\boxplus$ mitsubishi & 0 & \multicolumn{2}{|l|}{0,098} \\
\hline \multirow[t]{10}{*}{ Total Geral } & 0 & 0,069 & Total Geral & 0 & \multicolumn{2}{|l|}{0,098} \\
\hline & Car Category 1 & $\Sigma=\nabla_{x}$ & Car Category $2 \quad$ 测 & Car Category & 決 & $F_{x}$ \\
\hline & Economy & & Hatchback & Executive_Lu & & ^ \\
\hline & Family & & Family & Full_Size_Lux & & \\
\hline & Luxury & & Luxury & Mid_Size_Lu & & \\
\hline & Off_Roader & & Off_Roader & Sport_utility & & \\
\hline & Pickup & & Pickup & Pickup_Truc & & \\
\hline & Sedan & & Sedan & Crossover_S & & 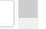 \\
\hline & Sport & & Sport & Full_Size_Sec & & \\
\hline & & & & Mid_Size_Se & & $\checkmark$ \\
\hline
\end{tabular}

Fig. 3. Emotional analysis

\begin{tabular}{|c|c|c|c|c|}
\hline Car Sentiment Score (entities) & Median_score & \multirow{2}{*}{\multicolumn{3}{|c|}{ Top 10 Best Keywords IT Median_score }} \\
\hline Evolvo & $\quad 0,520$ & & & \\
\hline & 0,520 & interior. great design & - & 0,937 \\
\hline Total Geral & 0.520 & dark grey car & - & 0,937 \\
\hline \multirow{2}{*}{\multicolumn{2}{|c|}{ Car Sentiment Score (Keywords) TrT Median_score }} & modern body design & & 0,934 \\
\hline & & comfortable seats & - & 0,917 \\
\hline Evolvo & $\quad 0,512$ & great driving experience. & & 0,902 \\
\hline \multirow{4}{*}{ Total Geral } & 0,512 & TS AWD & e & 0,899 \\
\hline & 0,512 & $\begin{array}{l}\text { interior design } \\
\text { enioyable car }\end{array}$ & : & 0,898 \\
\hline & & $\begin{array}{l}\text { enjovable car } \\
\text { attrartive looking car }\end{array}$ & : & 0,8962 \\
\hline & & Total Geral & & 0.912 \\
\hline Top 10 Worst keywords & 1T Median_score & Top 10 Worst Entities & \multirow{2}{*}{\multicolumn{2}{|c|}{ IT) Median_score }} \\
\hline unsuspecting Volvo & & Fuel_Economy & 0 & \\
\hline sporty manual transmission & $-0,599$ & Aceptable & 0 & \\
\hline tail fog light & 0,000 & Car_Type & - & 0,258 \\
\hline crash test ratings & 0.000 & Sound_System & - & 0.384 \\
\hline rain sensing wipers & 0,000 & Transmission & - & 0,400 \\
\hline $\begin{array}{l}\text { Volvo desilet } \\
\text { Veepo whara }\end{array}$ & 0,000 & Exterior & & 0,005 \\
\hline $\begin{array}{l}\text { Jeep Wrangler Sahara } \\
\text { volvo S40 TS }\end{array}$ & 0,000 & Build_quality & - & 0.469 \\
\hline tar & 0,111 & 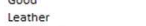 & - & 0,530 \\
\hline & 0,128 & acceleration & - & 0,583 \\
\hline Total Geral & -0.122 & Total Geral & 0 & 0.355 \\
\hline
\end{tabular}
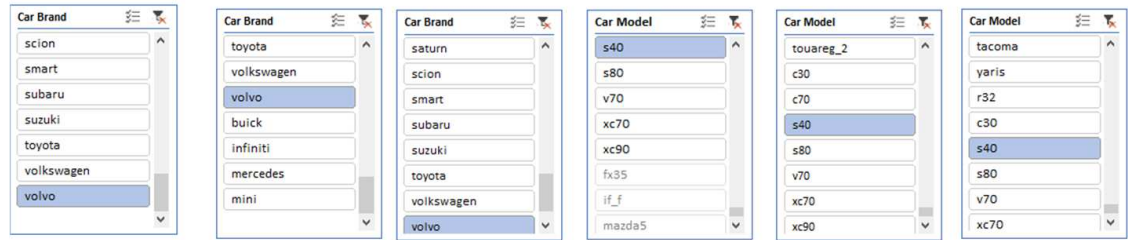

Fig. 4. Sentimental car analysis 
In the upper left corner of Figure 4, the score obtained from the sentimental analysis of the specific car model is shown, either from the Entities or the Keywords cube. It is a pretty good value, that means people got a good impression of the car model. In the three tables on the right, the "top 10 keywords", "top 10 entities" and "top 10 favorite entities" are shown. It is easy to check that people liked the interior, the seats, the acceleration and some other features. In the three tables below, "top 10 worst keywords", "top 10 worst entities" and "problems" are measured using the "median_score" that measures the sentiment. In these three tables referenced above, there are not many components referenced as negative, other than the transmission of the car, and as neutral the fuel consumption of the car.

In these different analyses presented above, the resulted obtained were quite satisfactory, since it was possible to extract relevant information about the cars as: sentimental and emotional analysis; what problems/weakness and components did people most like/strengths; made it possible to perform a deep analysis of a car model.

\section{Conclusions, Limitations and Future Work}

\subsection{Conclusions}

This paper proposed an experimental work to understand if it is possible to extract information insights from data variety, i.e., unstructured and semi-structured. It was quickly realized that to take advantage of unstructured and semi-structured data, it was necessary to find a way to transform and store them. For this, an architecture solution was defined with the objective of extracting useful information from unstructured and semi-structured data. Next, the architecture solution was evaluated and tested, which allowed to realize that it can extract relevant information from unstructured and semistructured data.

An analytical platform was developed to carry out different analyzes of the data obtained from the experiment. In the analytical platform, it was possible to verify a set of relevant information such as: sentimental and emotional analysis; what are the problems/strengths associated with a car; and a detailed analysis of a car.

\subsection{Limitations}

In this paper the following limitation is identified, the experiment created cannot be used as a generic solution. Consequently, for each specific case, it is necessary to calibrate the tools to the dataset available and to the results pretended.

\subsection{Future Work}

As future work, it will be relevant to carry out a study using unstructured and semistructured data in a real organization, to show that the data variety can reveal useful information's for analysis and, depending on data formats and types, explore other techniques of data analysis to understand what type of information can be obtained. 


\section{Acknowledgments}

This work has been supported by COMPETE: POCI-01-0145-FEDER-007043 and FCT - Fundação para a Ciência e Tecnologia within the Project Scope: UID/CEC/00319/2013.

\section{References}

[1] H. Baars and H.-G. Kemper, "Management Support with Structured and Unstructured Data—An Integrated Business Intelligence Framework," Inf. Syst. Manag., vol. 25, no. 2, pp. 132-148, Mar. 2008.

[2] T. Asai, K. Abe, S. Kawasoe, H. Arimura, H. Sakamoto, and S. Arikawa, "Efficient Substructure Discovery from Large Semi-structured Data," IEICE Trans. Inf. Syst., pp. 2754-2763, 2004.

[3] P. Russom, "BIG DATA ANALYTICS BIG DATA A N A LY TIC S TDWI BEST PRACTICES REPORT Introduction to Big Data Analytics," 2011.

[4] X. Dong et al., "Knowledge vault," in Proceedings of the 20th ACM SIGKDD international conference on Knowledge discovery and data mining - KDD '14, 2014, pp. 601-610.

[5] L. Li, Y. Yao, J. Tang, W. Fan, and H. Tong, "QUINT," in Proceedings of the 22nd ACM SIGKDD International Conference on Knowledge Discovery and Data Mining KDD '16, 2016, pp. 985-994.

[6] Y. Zhou, L. Liu, and D. Buttler, "Integrating Vertex-centric Clustering with Edgecentric Clustering for Meta Path Graph Analysis," in Proceedings of the 21th ACM SIGKDD International Conference on Knowledge Discovery and Data Mining - KDD '15, 2015, pp. 1563-1572.

[7] B. Bi, H. Ma, B.-J. (Paul) Hsu, W. Chu, K. Wang, and J. Cho, "Learning to Recommend Related Entities to Search Users," in Proceedings of the Eighth ACM International Conference on Web Search and Data Mining - WSDM '15, 2015, pp. 139-148.

[8] R. Blanco, G. Ottaviano, and E. Meij, "Fast and Space-Efficient Entity Linking for Queries," in Proceedings of the Eighth ACM International Conference on Web Search and Data Mining - WSDM '15, 2015, pp. 179-188.

[9] K. L. Caballero Barajas and R. Akella, "Dynamically Modeling Patient's Health State from Electronic Medical Records," in Proceedings of the 21th ACM SIGKDD International Conference on Knowledge Discovery and Data Mining - KDD '15, 2015, pp. 69-78.

[10] M. Kokkodis, P. Papadimitriou, and P. G. Ipeirotis, "Hiring Behavior Models for Online Labor Markets," in Proceedings of the Eighth ACM International Conference on Web Search and Data Mining - WSDM '15, 2015, pp. 223-232.

[11] N. Zalmout and M. M. Ghanem, "Multivariate adaptive community detection in Twitter," Int. J. Big Data Intell., vol. 3, no. 4, p. 239, 2016.

[12] T. P. Oliveira, J. S. Barbar, and A. S. Soares, "Computer network traffic prediction: a comparison between traditional and deep learning neural networks," Int. J. Big Data Intell., vol. 3, no. 1, p. 28, 2016.

[13] N. Makrynioti et al., "PaloPro: a platform for knowledge extraction from big social data and the news," Int. J. Big Data Intell., vol. 4, no. 1, p. 3, 2017.

[14] G. Dimitrakopoulos, V. Chatzigiannakis, and L. Tsitouras, "A knowledge-based integrated framework for increasing social management intelligence," Int. J. Big Data Intell., vol. 4, no. 1, p. 36, 2017.

[15] S. Mukherjee, G. Weikum, and C. Danescu-Niculescu-Mizil, "People on drugs," in 
Proceedings of the 20th ACM SIGKDD international conference on Knowledge discovery and data mining - KDD '14, 2014, pp. 65-74.

[16] V. Shashidhar, N. Pandey, and V. Aggarwal, "Spoken English Grading," in Proceedings of the 21 th ACM SIGKDD International Conference on Knowledge Discovery and Data Mining - KDD '15, 2015, pp. 2089-2097.

[17] Z. Chen and B. Liu, "Mining topics in documents," in Proceedings of the 20th ACM SIGKDD international conference on Knowledge discovery and data mining - KDD '14, 2014, pp. 1116-1125.

[18] S. Wang, Z. Chen, G. Fei, B. Liu, and S. Emery, "Targeted Topic Modeling for Focused Analysis," Proc. 22nd ACM SIGKDD Int. Conf. Knowl. Discov. Data Min. - KDD '16, pp. 1235-1244, 2016.

[19] T. Kurashima, T. Iwata, N. Takaya, and H. Sawada, "Probabilistic latent network visualization," in Proceedings of the 20th ACM SIGKDD international conference on Knowledge discovery and data mining - KDD '14, 2014, pp. 1236-1245.

[20] E. Schubert, M. Weiler, and H.-P. Kriegel, "SigniTrend," in Proceedings of the 20th ACM SIGKDD international conference on Knowledge discovery and data mining $K D D$ '14, 2014, pp. 871-880.

[21] M. Nagarajan et al., "Predicting Future Scientific Discoveries Based on a Networked Analysis of the Past Literature," in Proceedings of the 21 th ACM SIGKDD International Conference on Knowledge Discovery and Data Mining - KDD '15, 2015, pp. 20192028.

[22] B. Geerdink, "A reference architecture for big data solutions - introducing a model to perform predictive analytics using big data technology," Int. J. Big Data Intell., vol. 2, no. 4, p. 236, 2015.

[23] M. Papaioannou, E. Plum, E. T. F. Rogers, J. Valente, and N. I. Zheludev, "All-Optical Image Recognition Using Metamaterials," in Frontiers in Optics 2016, 2016, p. FF5G.7.

[24] K. Ganesan and C. Zhai, "Opinion-based entity ranking," Inf. Retr. Boston., vol. 15, no. 2, pp. 116-150, Apr. 2012. 